\title{
CIVILTÀ DEL LEGNO: IL MONASTERO
}

\section{PAOLA GALETTI}

UDC: 726.71

7.023.1-035.3

Preliminary communication

Manuscript received: 30. 10. 2016.

Revised manuscript accepted: 07. 02. 2017.

DOI: 10.1484/J.HAM.5.113726
P. Galetti

Dipartimento di Storia Culture Civiltà Alma Mater Studiorum Università di Bologna P.zza San Giovanni in Monte 2 40124, Bologna, Italy paola.galetti@unibo.it

The contribution would like to offer some reflections on use of wood in the monastic building structures, considering both written and archaeological sources. Attention is particularly directed to the articulation of the monastic space, to constructive activities and to material culture.

Keywords: monastic space, written sources, archaeological sources, wood, building construction, material culture

Il mio contributo, attraverso la considerazione di fonti scritte e di fonti materiali, vuole offrire una serie di riflessioni sulle strutture edilizie monastiche, su quelli che potremmo definire gli 'spazi dei monaci', con particolare riguardo, tra i materiali costruttivi, al legno e alla sua messa in opera, relativamente ai secoli V-XI e con lo sguardo rivolto alla penisola italiana ma anche Oltralpe. Oltre a ciò, cercherò per quanto possibile di proporre qualche considerazione anche sulla cultura materiale legata a questo materiale presente in ambiente monastico. Perciò, inevitabilmente, l'attenzione sarà rivolta anche all'attività lavorativa prevista e necessaria per edificazione e riattamento delle strutture e al personale impiegato per le diverse attività produttive.

Una prima osservazione da fare è relativa al problema della definizione dell'articolazione interna dello spazio monastico, dell'organizzazione degli ambiti nei quali i monaci trascorrevano la loro esistenza quotidiana. Le fonti scritte permettono di arrivare, anche se, soprattutto per i secoli dal IV al VII, in maniera non precisa ma indiretta, a una conoscenza per lo meno delle linee di sviluppo della progressiva definizione della conformazione dello spazio monastico, della topografia interna del monastero e di come funzioni diverse cui esso doveva assolvere (di tipo religioso, politico, produttivo, assistenziale, oltre che legate alla quotidianità pur sempre di un gruppo di uomini) si combinassero con la fruizione di una concreta realtà'.
D’altro lato l'archeologia dei monasteri tardo antichi e altomedievali, che a lungo si è identificata nella maggior parte dei casi con quella degli edifici di culto che li caratterizzavano, in primo luogo la chiesa abbaziale ${ }^{2}$, si è progressivamente rivolta negli ultimi anni, come ha sottolineato Eleonora Destefanis nel 2011, anche a nuovi aspetti, quali, tra l'altro, la morfologia delle strutture che ospitavano le attività quotidiane dei monaci e la loro dislocazione all'interno dell'impianto generale del monastero ${ }^{3}$. Sul piano metodologico, poi, l'affermazione dell'indagine stratigrafica ha consentito di precisare cronologie e fasi e ha anche - e questo per me è significativo - permesso di recuperare strutture in materiali deperibili, vedi appunto il legno, sia ascrivibili ai primi momenti di vita di una istituzione sia coesistenti con gli impianti in muratura, aprendo così lo sguardo sull'evoluzione delle tecniche costruttive, sui cantieri monastici e sulle attività artigianali ad essi deputate, oltre che sul personale in esse impiegato. $\grave{E}$ da notare, comunque, che per ambedue gli ambiti disciplinari possiamo notare una disparità nell'avanzamento degli studi in aree geografiche diverse, che limita certamente la possibilità di arrivare a una visione d'insieme.

Sul tema degli spazi monastici, a livello generale, la fase tardo antica e i primi momenti dell'altomedioevo risultano ancora scarsamente noti sul piano archeologico ${ }^{4}$, ma anche le fonti scritte sono spesso ambigue e di interpretazione problematica ${ }^{5}$. Più chiaramente la situazione si può delineare

${ }^{1}$ F. MARAZZI, Le città dei monaci. Storia degli spazi che avvicinano a Dio, Milano, 2015, p. 343-355.

${ }^{2}$ G. CANTINO WATAGHIN, Archeologia dei monasteri. L'altomedioevo, in S. Gelichi (a cura di), I Congresso Nazionale di Archeologia Medievale, Firenze, 1997, p. 265-268: 265; G.P. BROGIOLO, Dalla corte regia al monastero di San Salvatore. Le sequenze di scavo, in Id., F. Morandini (a cura di), Dalla corte regia al monastero di San Salvatore - Santa Giulia di Brescia, Mantova, 2014, p. 419-504.

3 E. DESTEFANIS, Archeologia dei monasteri altomedievali tra acquisizioni raggiunte e nuove prospettive di ricerca, in Post-Classical Archaeologies, 1, 2011, p. 349-382: 351 .

${ }^{4} \mathrm{G}$. CANTINO WATAGHIN, Istituzioni monastiche nel Friuli altomedievale: un'indagine archeologica, in Paolo Diacono e il Friuli altomedievale (secc.VI-X), I, Spoleto, 2001, p. 281-319:310; A. AUGENTI, Archeologia dell'Italia medievale, Roma-Bari, 2016, p. 105-106, 132-139, 182-184.

5 E. DESTEFANIS, Archeologia dei monasteri, op. cit. (n. 3), p. 351. Vedasi anche: H. R.Sennhauser (a cura di), Wohn-und Wirtschaftsbauten frühmittelalterlicher Klöster, Zürich, 1996; P. Racinet (a cura di), Pratique et sacré dans les espaces monastiques au Moyen Age et à l'époque moderne, Amiens, 1998; G. Spinelli (a cura di), Il monachesimo italiano dall'età longobarda all'età ottoniana (secc. VIII-X), Cesena, 2006. 
per i secoli dall'VIII all'XI, attraverso la lenta definizione di una forma codificata di monastero, con la chiesa abbaziale e i corpi di fabbrica ospitanti funzioni diverse disposti in un blocco compatto intorno al chiostro. Questo processo è ben delineato nel recente studio di Federico Marazzi del $2015^{6}$, a partire dalla considerazione di fonti scritte quali le Regole monastiche occidentali, nelle quali è difficile riscontrare l'indicazione di planimetrie ricorrenti, ad eccezione forse della cosiddetta Regula Isidori redatta tra 615-619 e attribuita a Isidoro di Siviglia7, per arrivare alla progettualità ideale per un insediamento monastico della Pianta di San Gallo risalente agli anni '3o del IX secolo, che trovò una compiuta realizzazione tra tardo secolo X e inizi dell'XI nell'abbazia di Cluny ${ }^{8}$. A queste testimonianze fanno da contrappunto i dati della ricerca sul campo. L'elemento che offre unità al complesso monastico viene ad essere nel corso del tempo il recinto, che ne delinea i confini verso l'esterno, al cui interno si possono individuare gli spazi ove si svolgono i momenti principali della vita comune (preghiera, oratorium, refezione, riposo), quelli deputati al lavoro intellettuale e manuale.

Se già questi sviluppi dell'organizzazione degli spazi monastici tra IV-V secolo e inizi XI possono essere tracciati muovendosi con difficoltà tra testimonianze a lungo reticenti, spesso non esaurienti per delineare un quadro compiuto, e soprattutto dovendosi giostrare su dati provenienti da aree diverse non sempre ricollegabili sincronicamente tra loro, pensiamo quanto possa essere maggiormente difficile ritrovare nella documentazione, di qualsiasi tipo, testimonianze sul tema al centro del mio specifico e circoscritto interesse in questa sede, cioè l'uso del legno, sia impiegato nelle strutture monastiche, sia nella cultura materiale. Ma procediamo con ordine.

Come si è detto, la contrapposizione tra vita monastica e vita nel 'mondo' era chiaramente indicata dalla presenza di limina monasterii, che racchiudevano uno spazio, quello in cui i monaci vivevano e pregavano, vietato (del tutto o solo parzialmente in sue parti) a coloro che non appartenevano alla comunità. Già nei testi più antichi delle Regulae si fa riferimento alla porta d'ingresso del cenobio, varco d'accesso che doveva essere ben sorvegliato, e allo janitor / porta(ra) rius, il portinaio, che si doveva occupare anche dei locali esterni al recinto 9 . La separatezza dal mondo trovava poi una articolazione maggiore, ad esempio, nell'idea di spazio monastico concepita da San Colombano (e descritta dal suo biografo Giona), per la quale esso era costituito da spazi concentrici: il primo era inviolabile e riservato solo ai monaci e alla preghiera; il secondo quello in cui si svolgevano le attività necessarie alla vita quotidiana dei monaci stessi, che poteva prevedere un contatto con il mondo esterno; il terzo era quello in cui poteva essere previsto l'incontro con il mondo dei laici ${ }^{10}$.

Se per il monastero di Jumièges, nella seconda metà del VII secolo, il biografo del suo fondatore, Filiberto, ci racconta che il santo costruì un recinto murario turrito a pianta quadrata, a delimitare quasi un castrum monastico" ${ }^{11}$, e se per il 'nuovo'(dopo il trasferimento) monastero di San Vincenzo al Volturno di XI secolo la ricerca archeologica ha evidenziato un muro di cinta dotato di torri angolari e affiancato da fossati ${ }^{12}$, diversa si presentava la situazione nel monastero

${ }^{6}$ F.MARAZZI, Le città dei monaci, op. cit. (n.1). Vedasi anche: C. Bertelli, G.P. Brogiolo (a cura di), Il futuro dei Longobardi. L'Italia e la costruzione dell'Europa di Carlo Magno. Saggi, Milano, 2000 (in particolare i saggi di M. DE JONG, P. ERHART, Monachesimo tra i Longobardi e i Carolingi, p. 105-127; G. CANTINO WATAGHIN, Monasteri tra VIII e IX secolo: evidenze archeologiche per l'Italia settentrionale, p. 129-141); G.M. CANTARELLA, Lo spazio dei monaci, in Uomo e spazio nell'alto medioevo, Spoleto, 2003, p. 805-854; H. DEY, Architettura monastica dagli inizi all'epoca carolingia, in S. de Blaaw (a cura di), Storia dell'architettura italiana. Da Costantino a Carlo Magno, Milano, 2010, p. 300-321; H. Dey, E. Fentress (a cura di), Medieval Monasticism Ante Litteram. The Spaces of Monastic Observance in Late Antiquity and the Early Middle Ages, Turnhout, 2011.

${ }^{7}$ Regola di Isidoro, in E. Bianchi (a cura di), Regole monastiche d'Occidente, Torino, 2001, p. 305-336: 308.

${ }^{8}$ H.R. SENNHAUSER, St. Gallen. Klosterplan und Gozbertbau. Zur Rekonstruktion des Gozbertbaues und zur Symbolik des Klosterplanes, Zürich, 20o1; ID., St. Gallen: zum Verhältnis von Klosterplan und Gozbertbau, in Hortus artium medievalim (= HAM), 8, 2002, p. 49-56; A. BAUD, L'abbaye de Cluny à travers ses plans, in Cluny ou la puissance des moines. Histoire de l'abbaye et de son ordre, 910-179o, in Dossiers d'Archéologie, 269, 2002, p. 80-81; B. BRENK, La pianta del monastero di San Gallo e la regola di San Benedetto, in R. Cassanelli, E. Lopez-Tello Garcia (a cura di), Benedetto e l'eredità artistica, Milano, 2007, p. 73-80; H.R. SENNHAUSER, À propos de l'architecture monastique entre Saint-Gall et Cluny II, in D. Iogna-Prat et alii (a cura di), Cluny. Les moines et la société au premier âge féodal, Rennes, 2013, p. 527-547; G. CANTINO WATAGHIN, Cantieri monastici nell'alto medioevo in Italia settentrionale, in M.C. Somma (a cura di), Cantieri e maestranze nell'Italia medievale, Spoleto, 2010, p. 279-344; F.J. MORENO MARTIN, La arquitectura monastica hispana entre la Tardoantiguedad y la alta Edad Media, Oxford, 2011; G. ARCHETTI, "Dilexi decorem domus tuae". Committenza aristocratica e popolare in ambito claustrale (secoli VIII-XII), in A.C. Quintavalle (a cura di), Il Medioevo: i committenti, Atti del Convegno internazionale di studi (Parma, 21-26 settembre 2010), Milano, 2011 (I convegni di Parma, 13), p. 237-251; ID., Spazi e strutture claustrali nei commenti carolingi alla Regola benedettina, in HAM, 20, 2, 2014, p. 448-462; ID., Boscose solitudini. Simboli, immagini e figure dal mondo monastico, in A.C. Quintavalle (a cura di), Medioevo: natura e figura. La raffigurazione dell'uomo e della natura nell'arte medievale, XIV Convegno internazionale di studi (Parma, 20-25 settembre 2011), Milano, 2015 (I convegni di Parma, 14), p. 169-182; A. BAUD, CH. SAPIN, Les fouilles de Cluny: état des recherches recentes sur les débuts du monastère et ses églises. Cluny I et Cluny II, in D. Iogna-Prat et alii (a cura di), Les moines, op. cit. (n. 8), p. 497-514; F. MARAZZI, Le città dei monaci, op. cit. (n. 1), p. 280-295, 303-338. Vedasi inoltre il sito: http://www.stgallplan.org/index.htlm.

${ }_{9}^{2}$ Regola dei Quattro Padri, in E. Bianchi (a cura di), Regole monastiche d'Occidente, op. cit. (n. 7), p. 29-40: 35-36; Regola Orientale, in Ibid., p. 61-79: 73; A. De Vogüé (éd.), Regula ad monachos (La Règle du Maître), Paris, 1964-1965, XCV. Vedasi anche: E. DESTEFANIS, Ad portam monasterii. Accessi e spazi limitanei nei monasteri dell'Occidente altomedievale (secoli VI-IX), in Ead., C. Lambert (a cura di), Per diversa temporum spatia. Scritti in onore di Gisella Cantino Wataghin, Torino, 2011, p. 51-84.

${ }^{10}$ Vitae Columbani abbatis discipulorumque eius libri duo auctore Iona, ed. B. Krusch, in Passiones Vitaeque Sanctorum Aevi Merovingici, II, Hannoverae et Lipsiae, 1902 (MGH, Scriptores Rerum Merovingicarum, IV), p. 1-152: 19, 20. Vedasi anche: E. JAMES, Archaeology and the Merovingian Monastery, in H.B. Clarke, M. Brennan (a cura di), Columbanus and Merovingian Monasticism, Oxford, 1981, p. 33-58; F. MARAZZI, Le città dei monaci, op. cit. (n. 1), p. 128-129.

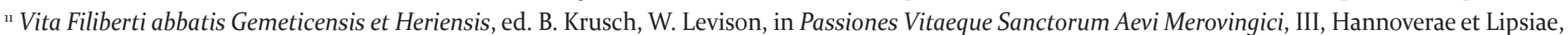
1910 (MGH, Scriptores Rerum Merovingicarum, V), p. 568-604, 8. Vedasi anche: N. MANOURY, Les bâtiments conventuels de l'abbaye de Jumièges, in Archéologie médiévale, 26, 1997, p. 77-107; J. LE MAHO, L'abbaye de Jumièges, Paris, 2001; ID., Le monastère de Jumièges aux temps Mérovingiens (VII'-VIII ${ }^{e}$ siècle): les témoignages des testes et de l'archéologie, in HAM, 9, 2003, p. 315-322.

${ }^{12}$ Di grande utilità per uno sguardo d'insieme: F. Marazzi (a cura di), San Vincenzo al Volturno. Guida alla città monastica benedettina, Cerro al Volturno (IS), 2014, p. 53-54. Su San Vincenzo al Volturno, dello stesso autore, tra l'altro: ID., San Vincenzo al Volturno: evoluzione di un progetto monastico fra IX 
femminile di Hamage, fra VII e VIII secolo, situato nella regione nord-orientale del regno franco, attuale Pas-de-Calais francese. Qui gli scavi degli anni '8o-'9o del secolo scorso hanno evidenziato un recinto costituito da una palizzata lignea - e questo ci interessa - affiancata all'esterno da un fossato ${ }^{13}$. Così pure, se ci spostiamo in Italia, e precisamente a Bobbio, la biografia dell'abate Attala, successore di san Colombano fra 615-627, ci mostra un complesso delimitato da un recinto definito saepta, termine di per sé ambiguo, che però può essere inteso o come semplice limite o come indicazione di un limite rappresentato da un diaframma vegetale $e^{14}$.

Al recinto dell'abbazia piacentina, ma senza precisazioni sulla sua realtà materiale, fa pure riferimento il Breve memorationis in cui l'abate Wala, tra 833 e 835, regolò l'organizzazione del complesso monastico ${ }^{15}$. Se non di recinti in legno, abbiamo però attestazioni dell'esistenza di fossati a individuare l'area riservata ai monaci dagli scavi effettuati a Nonantola, Sesto al Reghena, Leno ${ }^{16}$. Un dato significativo sugli apprestamenti lignei legati all'accesso al monastero ci viene dagli scavi di San Vincenzo al Volturno. Nel IX secolo si accedeva al cenobio attraverso un sistema di pontili lignei, che si protendevano nel letto del fiume. Resti di queste strutture, interessate dall'incendio arabo dell'881, sono stati indagati nell'area antistante la chiesa sud e il cosiddetto refettorio degli ospiti; reinterrati, non sono oggi più visibili. Si trattava di un piano realizzato con tavolati lignei che poggiavano su di un sistema di pali di legno o di blocchi di pietra infissi nel letto del fiume. Nel tratto esplorato il tavolato era unito a un molo in muratura collegato a un ingresso che immetteva nel refettorio degli ospiti. Lo scavo ha permesso anche di evidenziare che la facciata degli edifici monastici fondata entro il letto del fiume era stata protetta da file di pali di legno che la riparavano dall'erosione causata dalla corrente ${ }^{17}$.

All'interno del recinto, secondo modalità inizialmente più destrutturate, poi via via organizzate secondo una planimetria codificata o per lo meno preferita per la sua razionalità a partire dal X secolo e mutuata dalla Pianta di San Gallo, con individuazione di una clausura monastica e di aree aperte agli esterni, si disponevano i locali della vita comunitaria e corpi di fabbrica dalle funzioni diversificate: la chiesa abbaziale, innanzitutto, poi anche altre diverse chiese/cappelle, anche con funzione funeraria per la comunità ${ }^{18}$, chiostro, sala capitolare, refettorio, dispense/cellari, cucine, forno, cantine, infermeria, bagni, latrine, magazzini, stalle, mulino, celle singole o dormitorio comune, scriptorium, biblioteca/ archivio, opifici legati ad attività produttive, foresteria, oltre a un orto/frutteto. Il tutto a ricreare un microcosmo di fatto autonomo, all'interno del quale dovevano essere soddisfatte in primo luogo le esigenze dell'anima, ma anche quelle del corpo. San Benedetto, infatti, nel capitolo LXVI della Regola dice: «Il monastero, se è possibile, deve essere organizzato in modo che per tutto ciò che è necessario - cioè l'acqua, il mulino, l'orto e i vari mestieri che vengono esercitati all'interno del monastero - i monaci non abbiano bisogno di andare in giro fuori, poiché ciò non giova assolutamente alle loro anime» ${ }^{19}$.

È utile a questo punto concentrare l'attenzione sui materiali costruttivi delle diverse strutture edilizie, per rintracciare testimonianze sull'impiego del legno. Una considerazione va fatta. Le fonti scritte forniscono infor-

e XI secolo, in G. Spinelli (a cura di), op. cit. (n. 5), p. 425-46o; ID., San Vincenzo al Volturno. L'impianto architettonico fra VIII e XI secolo, alla luce dei nuovi scavi della basilica maior, in F. De Rubeis, Id. (a cura di), Monasteri in Europa occidentale (secoli VIII-XI): topografia e strutture, Roma, 2008, p. 323-390; ID., Varcando lo spartiacque. San Vincenzo al Volturno dalla fondazione alla conquista franca del regnum langobardorum, in V. Pace (a cura di), L'VIII secolo. Un secolo inquieto, Cividale del Friuli, 2010, p. 163-184; ID., San Vincenzo al Volturno dal X al XII secolo. Le "molte vite" di un monastero fra poteri universali e trasformazioni geopolitiche del Mezzogiorno, Roma, 2011. Vedasi anche: R. Hodges (a cura di), San Vincenzo al Volturno I. The 1980-1986 Excavations, Roma-London, 1993 e Id. (a cura di), San Vincenzo al Volturno II. 1980-1986 Excavations, Roma-London, 1995 (The British School at Rome Archaeological Monographs, 5, 7); F. Marazzi (a cura di), San Vincenzo al Volturno. Cultura, istituzioni, economia, Montecassino, 1996; P. DELOGU, R. HODGES, J. MITCHELL, San Vincenzo al Volturno. La nascita di una città monastica, Castel San Vincenzo, 1996; R. HODGES, Light in the Dark Ages. The Rise and Fall of San Vincenzo al Volturno, London, 1997; J. Mitchell, I.L. Hansen (a cura di), San Vincenzo al Volturno III: the Finds from the 1980-86 Excavations, Spoleto, 2001; R. HODGES, S. LEPPARD, J. MITCHELL, San Vincenzo Maggiore and hits Workshops, London, 2011; IDD., Reconstructing the Later Eighth-Century Claustrum at San Vincenzo al Volturno, in Papers of the British School at Rome, 80, 2012, p. 145-155.

${ }^{13}$ É. LOUIS, Hamage (Nord). Ėspaces et bâtiments d'un monastère mérovingien et carolingien, in P. Racine (a cura di), Pratique et sacré dans les éspaces monastiques au Moyen-Âge, op. cit. (n. 5), p. 73-97; ID., Sorores et fratres in Hamatico degentes. Naissance, évolution et disparition d'une abbaye au Haut Moyen-Âge: Hamage (France, Nord), in Les monastères mérovingiens et leurs domaines, De la Meuse à l'Ardenne, 29, 1999, p. 15-47; ID., A de- romanised Landscape in Northern Gaul: the Scarpe valley from the 4 th to the gth Century AD, in W. Bowden, L. Lavan (a cura di), Recent Research on the Late Antique Countryside, Leiden, 2004, p. 479-504.

${ }^{14}$ E. DESTEFANIS, Il monastero di Bobbio in età medievale, Firenze, 2002, p. 36, n. 20; F. MARAZZI, Le città dei monaci, op. cit. (n. 1), p. 153.

${ }^{15}$ C. Cipolla (a cura di), Codice Diplomatico del monastero di San Colombano di Bobbio, I, Roma, 1918, XXXVI, p. 139-141.

${ }^{16} \mathrm{~S}$. GELICHI, Il monastero di Nonantola e le sue terre. Controllo del territorio e organizzazione dell'insediamento nel medioevo, in L. Pani Ermini (a cura di), Committenza, scelte insediative e organizzazione patrimoniale nel medioevo, Spoleto, 2007, p. 325-355: 342; ID., M. LIBRENTI, Ricerche archeologiche su una grande abbazia altomedievale: San Silvestro di Nonantola, in J. Lopez Quiroga, A.M. Martinez Tejera, J. Morin de Pablos (a cura di), Monasteria et Territoria. Élites, edilicia y territorio en el Mediterraneo medieval (siglos V-XI), Oxford, 2007, p. 337-348: 340; G.C. Menis, A. Tilatti (a cura di), L'abbazia di Santa Maria di Sesto fra archeologia e storia, Fiume Veneto, 1999; E. DESTEFANIS, M. LAUDATO, S. VITRI, Nuove indagini archeologiche all'abbazia di Sesto al Reghena (PN), in R. Fiorillo, P. Peduto (a cura di), III Congresso Nazionale di Archeologia Medievale, Firenze, 2003, p. 206-212; A. BREDA, L'indagine archeologica nel sito dell'abbazia di S. Benedetto di Leno, in A. Baronio (a cura di), San Benedetto "ad Leones" un monastero benedettino in terra longobarda, Brescia, 2006 (Brixia sacra, XI, 2), p. 111-139: 139.

${ }^{17}$ F. Marazzi (a cura di), San Vincenzo al Volturno. Guida, op. cit. (n. 12), p. 46-47; F. MARAZZI, Iuxta flumen Vulturnum. Gli scavi lungo il fronte fluviale di San Vincenzo al Volturno, Cerro al Volturno (IS), 2015.

${ }^{18}$ Sulla moltiplicazione dei poli cultuali all'interno del recinto monastico: E. DESTEFANIS, Archeologia dei monasteri altomedievali, op. cit. (n. 3), p. 366360 con casistica e relativi riferimenti bibliografici.

${ }^{19}$ Regola di Benedetto, in E. Bianchi (a cura di), Regole monastiche d'Occidente, op. cit. (n. 7), p. 193-264: LXVI, 26o; S. BENEDICTI, Regula, in S. Pricoco (a cura di), La Regola di San Benedetto e le Regole dei Padri, Milano, 1995, p. 114-385. Si veda inoltre: A. GALDI, Benedetto, Bologna, 2016, p. 36-50. 
mazioni disparate sui caratteri materiali dell'edilizia monastica e la ricerca archeologica si trova di fronte al fatto che per la sua natura di materiale organico il legno costituisce un problema, perché sopravvive raramente nei depositi sepolti di natura aerobica. Inoltre bisogna tenere presente che spesso ci troviamo di fronte a complessi rimaneggiati nel corso del tempo e che hanno pertanto spesso assistito ad un consapevole abbandono degli impianti originari in materiale deperibile.

Se la Vita di San Gallo, scritta da Wettinus, sembra rimandare ad un insediamento monastico composto da strutture, sia ecclesiastiche sia residenziali, dimesse sotto il profilo materiale per il VII secolo (sua morte nel 640), che non risultano avere subito importanti rimaneggiamenti fino alla metà dell'VIII secolo ${ }^{20}$, gli scavi del monastero di Hamage ci mostrano uno spazio delimitato, lo si è detto, da una palizzata lignea e un fossato, con all'interno capanne di legno e paglia che costituivano le cellulae delle monache. Verso la metà del secolo VIII una ristrutturazione portò alla edificazione di una chiesa in pietra e alla sostituzione delle singole celle con un edificio comunitario. C'è da dire però che solo tra XeXI secolo, in seguito ad un incendio (per scorrerie vichinghe?) furono obliterate le strutture lignee del chiostro carolingio, la cui traccia risulta dalla lettura stratigrafica ${ }^{21}$. Un complesso di edifici lignei disposti a quadrilatero attorno a uno spazio centrale aperto costituiva il monastero dei santi Ulrich e Afra, vicino ad Augusta in Baviera, intorno alla metà del VII secolo, ristrutturato nell'VIII con la sostituzione del legno con la pietra ${ }^{22}$.

L'uso del legno per la costruzione del primo impianto dei monasteri doveva essere una realtà, sfruttando risorse ampiamente disponibili o anche materiale di recupero, come nel caso del monastero di Redon, ai confini della Bretagna, per il quale i primi edifici destinati ad accogliere la comunità furono costruiti utilizzando il legname recuperato dallo smantellamento della casa di un benefattore, trasportato dagli stessi monaci sino al luogo prescelto ${ }^{23}$. Interessanti sono le testimonianze relative all'abbazia di Fontenelle, fondata da Wandregisilus in età merovingia non lontano da Rouen, restaurata alla fine del secolo VIII e ampiamente rimaneggiata da Ansegiso, abate dall'822 all'832, fortemente legato alla corte carolingia. È la fonte scritta, i Gesta Abbatum Fontanellensium, che descrive i lavori svolti, a fronte della mancanza di dati materiali riferibili alla cronologia indicata. Tra di essi va segnalata la costruzione del dormitorium fratruum nobilissimum, un ampio edificio «di duecento e otto piedi di lunghezza, ventisette di larghezza e sessantaquattro di altezza; i suoi muri furono costruiti con calce tenacissima..., sabbia rossastra e pietra tufacea fossile... All'interno era diviso in altezza da un solaio, il cui pavimento [del primo piano] era magnificamente decorato e che al di sopra aveva un soffitto ornato... con pitture. Il piano superiore dell'edificio aveva finestre in vetro e tutta la sua struttura, ad eccezione delle parti in muratura, era stata realizzata con legno di quercia di grande durevolezza..., inoltre, aveva travi a sostenere sia il soffitto sia il solaio». Gli stessi materiali costruttivi erano indicati per il refettorio ( «l'edificio era stato diviso a metà da un muro, cosicché una parte fungesse da refettorio e l'altra da dispensa. Le sue dimensioni e il materiale con cui fu costruito erano gli stessi del dormitorio») e sul culmine della torre della chiesa abbaziale dedicata a san Pietro, con una «piramide quadrangolare alta trentacinque piedi, costruita con elementi lignei scolpiti... coperta di piombo, stagno e rame dorato» ${ }^{24}$.

È inevitabile fare a questo punto una osservazione. Se abbiamo fornito esempi di impiego del legno come materiale costruttivo per le prime fasi di vita dei monasteri, sostituito successivamente con la pietra, dobbiamo tenere sempre presente che il legno continuò a lungo a costituire il materiale degli elementi accessori degli edifici, come scale, tramezzi, solai, ossature di tetti, anche copertura degli stessi. Considerazioni analoghe possiamo fare se ci spostiamo nella penisola e in particolare a San Vincenzo al Volturno, importantissimo caso di studio.

Se pochissimo si conosce sul suo impianto nei primi decenni di vita, a causa della preponderanza delle strutture della fase carolingia, possiamo però pensare che inizialmente potesse avere una struttura architettonica 'leggera' realizzata con materiali lapidei di recupero e con materiali deperibili, come legno, argilla, paglia. Abbiamo traccia del legno usato come elemento accessorio (frammenti di travature dei tetti, tramezzi, stipiti, assiti pavimentali, pali di sostegno...) attraverso lo scavo nelle aree danneggiate dall'incendio arabo dell'881: cucine, refettorio, ambiente attiguo alla corte a giardino. Gli scavi del 1983-84, ad esempio, hanno verificato tra i resti dell'incendio che devastò del tutto l'edificio del refettorio le travature del tetto in legno e anche al di sopra delle stesse i resti dell'ordito di una infrascatura che doveva servire a trattenere il calore. Interessanti per noi sono i dati recuperati nell'area delle cucine, suddivisa in due ambienti, la cucina vera e propria e un vano anticucina. L'incendio ci ha restituito il loro aspetto alla fine del IX secolo. L'anticucina, a pianta rettangolare ( $\mathrm{m} 8,30 \times \mathrm{x}$ ), aveva un tetto in legno sostenuto al centro da un palo e da una colonna. Sul lato sinistro rispetto all'entrata sono stati trovati i resti carbonizzati di una sorta di sgabuzzino-armadio. Poco sotto il suolo correva una canaletta la cui copertura era stata realizzata con un doppio tavolato ligneo costituito da piccole assi disposte trasversalmente. Il tavolato più profondo era appoggiato ai bordi originari della canaletta, mentre quello più superficiale

\footnotetext{
${ }^{20}$ Vita Galli confessoris triplex. Vita auctore Wettino, ed. B. Krusch, in Passiones Vitaeque, op. cit. (n. 10), p. 256-28o: I, 6; II, 6-8.

${ }^{21}$ E. DESTEFANIS, Archeologia dei monasteri, op. cit. (n. 3), p. 375-376. Sul monastero di Hamage: É. LOUIS, J. BLONDIAUX, L'abbaye mérovingienne et carolingienne de Hamage (nord). Vie, mort et sépulture dans une communauté féminine, in A. Alduc-Le Bagousse (a cura di), Inhumation de prestige ou prestige de l'inhumation? Expressions du pouvoir dans l'au-delà (IVe-XVe siècle), Caen, 2009, p. 117-149: 122-123; É. LOUIS, Archéologie en Nord-Pas-de-Calais. Wandignies-Hamage, ancienne abbaye de Hamage, s.l., 2002; la bibliografia alla nota 13.

${ }^{22}$ H. DANNHEIMER, Das coenobium beatae Afrae in Augsburg, in H.R. Sennhauser (a cura di), Wohn-und- Wirtschaftsbauten frühmittelalterlicher Klöster, op. cit. (n. 5), p. 33-46.

${ }^{23}$ J.M.H. SMITH, Aedificatio sancti loci: the making of a ninth-century holy place, in M.D.E Jong, F. Theuws, C. Van Rhijn (a cura di), Topographies of power in the Early Middle Ages, Leiden, 2001, p. 361-396: 361-363.

${ }^{24}$ Gesta Abbatum Fontanellensium, ed. S. Löwenfeld, Hannoverae, 1886, (MGH, Scriptores Rerum Germanicarum in usum Scholarum, 28), 16, 17; G.A. SIMON, L'abbaye de Saint-Wandrille, Grenoble, 1937; J. LAPORTE, Fontenelle ou St.-Wandrille, in R. Aubert (a cura di), Dictionnaire d'histoire et géographie ecclésiastique, 17, Paris, 1971, c. 915-953; F. MARAZZI, Le città dei monaci, op. cit. (n. 1), p. 267-271.
} 
era poggiato su assi poste sopra i conci di rialzamento delle spallette laterali. Parti di questo sistema di copertura sono state rinvenute in situ carbonizzate.

Nella cucina vera e propria è stata trovata poi una botola da cui si attingeva l'acqua dal fiume, che doveva essere coperta da un assito che poggiava su quattro travi lignee, sostenute da altrettanti piedritti costituiti da conci di travertino. Di queste travi è stata rinvenuta quasi integra quella sul lato verso il fiume; è di legno di castagno, è lunga circa 4 metri e presenta, sulla faccia superiore, un incasso per appoggiare le travi dell'assito. Sempre per la cucina, nella parte frontale del focolare è stata rinvenuta una buca, spia indiretta della presenza di un palo che, inserito diagonalmente nel terreno, doveva servire come base per appendere i contenitori per la cottura. Da ultimo vorrei segnalare che nell'area degli ambienti sotto il fianco est del colle della torre, un spazio pesantemente danneggiato dall'incendio dell' 881 al di sotto dei crolli ha presentato resti carbonizzati delle intelaiature lignee dei tetti e di una serie di tramezzi che ne suddividevano lo spazio interno. Travi di legno dovevano poi servire come rinforzo agli angoli delle murature in tecnica mista di un vano nel complesso delle officine. Si è potuto appurare che le principali essenze lignee utilizzate erano la quercia, il castagno e l'abete, recuperabili in loco $^{25}$.

Sui materiali da costruzione degli impianti monastici vorrei ora prendere in considerazione il caso bobbiese, attraverso la fonte scritta, in quanto l'area del monastero medievale risulta fortemente rimaneggiata da interventi ascrivibili a diverse epoche, ad iniziare dall'età rinascimentale, per cui manchiamo di fatto di dati materiali significativi riferiti al suo impianto. È la tradizione agiografica, pur con tutti i limiti di una fonte di questo tipo, che ci viene in soccorso: in primis la Vita di San Colombano, il fondatore del monastero intorno al 613 in val Trebbia nel Piacentino, scritta da Giona, quella del suo successore alla guida del cenobio, Attala (muore nel 627), redatta sempre da Giona, e i Miracula sancti Columbani, opera del X secolo ${ }^{26}$. La Vita di Colombano ci parla della edificazione del monastero nelle solitudini delle valli appenniniche, laddove c'era una basilica dedicata a San Pietro in rovina. «Il beato Colombano andato colà e trovata... la basilica semidiroccata, la restaurò... Durante tale restaurazione apparvero mirabili prodigi del Signore. Infatti si tagliavano tronchi d'abete in mezzo a scogli e a rocce scoscese, da dove era impossibile il trasporto coi carri. Ebbene: l'uomo di Dio si recava in quei luoghi con i suoi e, in due o tre, come permetteva il sentiero strettissimo, prendevano sulle spalle... dei pesi immani; a volte un tronco che sulla terra piana non sarebbero riusciti a trasportare... neppure trenta o quaranta persone... L'uomo di Dio, vedendo pertanto un aiuto così straordinario, esortò i suoi a condurre a termine in letizia l'opera incominciata... Restaurò quindi il tetto della basilica, le sommità dei muri in rovina e si preparò a costruire tutti quegli edifici che erano necessari a un monastero ${ }^{27}$.

D’altronde, di questa operosità legata al reperimento e utilizzo del legno da parte dei monaci la stessa Vita aveva già raccontato facendo riferimento a un miracolo del santo avvenuto presso il cenobio di Luxeuil, sempre di sua fondazione, laddove si narrava di un 'incidente sul lavoro' capitato ad un monaco mentre «con cunei e colpi di mazza», insieme ai confratelli, spaccava un tronco di quercia ${ }^{28}$. La Vita di Attala ci parla di un'opera di restauro compiuta dall'abate, comprendente anche le coperture dei tetti, tegumenta renovat, mentre i Miracula confermano l'impiego del legno per la prima fase costruttiva del monastero allorché magnificano i meriti dell'abate Agilulfo, al quale si attribuisce la ricostruzione in pietra (ex lapidibus struxit) della chiesa precedente, realizzata da Colombano ex lignis $^{29}$. La testimonianza va oltre certamente all'evidente intento agiografico di celebrazione dell'abate Agilulfo come innovatore. Se consideriamo la scansione cronologica delle fonti testé considerate, quei solarias VI, casas terraneas XXX inventariati per il complesso monastico nell'Adbreviatio dell'862 (e ricordate le sole XXX terraneas in quella dell'883) erano dunque in pietra, in legno $\mathrm{o}$ in tecnica mista ${ }^{30}$ ? D'altronde, nel quadro della menzione di forme di artigianato rurale, dobbiamo tenere presente che sempre in ambedue gli inventari dell'862 e dell'883 dei beni monastici, per la corte di Luliatica (nel comune di Santa Maria della Versa, nel Pavese) sono registrati sulla pars massaricia 7 fictales che tra le prestazioni dovute al monastero «cooperiunt casas cum suo ligno»"31.

A questo punto brevemente si può prendere in considerazione il tema degli artigiani che a vario titolo lavoravano il legno, in primis coloro che lo mettevano in opera nelle strut-

\footnotetext{
${ }_{25}$ F. Marazzi (a cura di), San Vincenzo al Volturno. Guida, op. cit. (n. 12), p. 81, 87, 90, 138-139; F. MARAZZI, I locali della refezione e le cucine dell'abbazia di San Vincenzo al Volturno nel IX secolo: i dati archeologici, in Id., A. Gobbi (a cura di), Il lavoro nella Regola. L'approvvigionamento alimentare e il cantiere edile di San Vincenzo al Volturno fra IX e XI secolo, Napoli, 2007, p. 27-34; A. GOBBI, Materiali e tecniche costruttive di un grande cantiere altomedievale, in Ibid., p. 83-212; A. CARANNANTE et alii, Le cucine di San Vincenzo al Volturno. Ricostruzione funzionale in base ai dati topografici, strutturali, bioarcheologici e chimici, in F. De Rubeis, F. Marazzi (a cura di), Monasteri in Europa occidentale, op. cit. (n. 12); la bibliografia citata alla nota 12. Sul materiale lapideo: A. GOBBI, San Vincenzo al Volturno. Approvvigionamento e lavorazione del materiale lapideo delle murature, in P. Galetti (a cura di), Edilizia residenziale tra IX-X secolo. Storia e Archeologia, Firenze, 2010, p. 153-169.

${ }^{26}$ Vita Columbani abbatis, op. cit. (n. 10); Ibid., II, 5; Miracula sancti Columbani, ed. H. Bresslau, Lipsiae, 1934 (in MGH, Scriptores, XXX/II), p. 993-1015; A. Dubreucq, A. Zironi (a cura di), Miracula sancti Columbani. La reliquia e il giudizio regio/ La relique et le jugement royal/ Relic and Royal Judgment, Firenze, 2015.

${ }^{27}$ Vita Columbani abbatis, op.cit. (n. 10), p. 107; GIONA, Vita di san Colombano. Versione italiana dal testo originale, E. Cremona, M. Paramidani (a cura di), Piacenza, 1965, p. 58-59.

${ }^{28}$ Vita Columbani abbatis, op. cit. (n. 10), p. 81; GIONA, Vita di san Colombano, op. cit. (n. 27), p. 26.

${ }^{29}$ E. DESTEFANIS, Il monastero di Bobbio, op. cit. (n. 14), p. 37; Ibid., p. 63; Miracula sancti Columbani, op. cit. (n. 26), I, p. 998. Vedasi inoltre: V. POLONIO, Il monastero di San Colombano di Bobbio dalla fondazione all'epoca carolingia, Genova, 1962; A. PIAZZA, Monastero e vescovado di Bobbio (dalla fine del X agli inizi del XIII secolo), Spoleto, 1997; F.G. Nuvolone (a cura di), La fondazione di Bobbio, Bobbio, 200o; A. ZIRONI, Il monastero longobardo di Bobbio. Crocevia di uomini, manoscritti e culture, Spoleto, 2004; R. CONVERSI, E. DESTEFANIS, Bobbio e il territorio piacentino tra VI e VII secolo: questioni aperte e nuove riflessioni alla luce dei dati archeologici, in Archeologia medievale, XLI, 2014, p. 289-312.

${ }^{30}$ Inventari altomedievali di terre, coloni e redditi, a cura di A. Castagnetti et alii, Roma, 1979: S. Colombano di Bobbio, ed. A. Castagnetti, I, a. 862, p. 127; II, a. 883 , p. 148.

${ }^{31}$ Ibid., p. 137, 158 .
} 
ture edilizie. Una testimonianza di grande interesse è fornita dal già ricordato Breve memorationis redatto dall'abate di Bobbio Wala fra 833 e 835 , che si proponeva di organizzare al meglio, secondo un ordine razionale, le risorse e anche le competenze tecniche di un grande monastero. Il Breve si apre con un elenco di tre gruppi di proprietà fondiarie, le cui rendite alimentavano altrettante voci del bilancio generale dell'abbazia: risorse per il victus fratruum, per la camara fratruum, per le ceterae necessitates. Poi passa ad elencare i ministeria quae infra monasterio agantur, cioè le mansioni da attivarsi nella comunità, con l'indicazione del responsabile, che di fatto corrispondevano a luoghi ben precisi nei quali dovevano essere svolte. Il testo è quindi utile testimonianza, anche se indiretta, dell'articolazione spaziale e funzionale del monastero. Al camararius abbatis toccava, tra l'altro, la supervisione delle officine dei bottai, dei mobilieri e dei muratori specializzati nella costruzione di mulini, case e muri in genere, mentre il magister carpentarius coordinava muratori, lapicidi e falegnami.

È significativa questa preminenza assegnata a chi ha competenze specialistiche nella lavorazione e messa in opera del legno rispetto agli altri artigiani delle costruzioni ${ }^{32}$. Il Breve rispecchia l'impostazione delle Consuetudines Corbeienses, emanate dall'abate di Corbie Adalardo (751-822), che dal 781 fino alla sua morte aveva retto il monastero della Francia del Nord, a est di Amiens. Qui, il lavoro e probabilmente lo spazio ad esso deputato era suddiviso in tre camarae: nella terza erano riuniti gli incaricati del taglio della legna, coloro che dovevano portarla al forno del pane e all'ingresso principale del monastero, i falegnami e i muratori e altrij3. Wala, d'altronde, era fratello di Adalardo, era stato monaco e anche abate a Corbie fra 826-830. Anche in questo caso merita attenzione l'importanza assegnata ai vari utilizzi del materiale ligneo. La complessità della lavorazione e messa in opera di molteplici materiali da costruzione necessari per l'edificazione e il buon mantenimento delle fabbriche monastiche, fra i quali il legno, come si è visto, è stata sottolineata anche dalle ricerche su San Vincenzo al Volturno ${ }^{34}$. Nell'area degli ambienti sotto il fianco est del colle della torre, nel vano definito come 'magazzino' sono stati ritrovati, oltre a vari resti carbonizzati di intelaiature lignee dei tetti e di tramezzi parimenti lignei, anche materiali che dovevano ancora essere messi in opera e utensili da muratore e da carpentiere ${ }^{35}$.
Le attività legate all'approvvigionamento del legno (scelta, taglio della materia) e alla sua messa in opera da solo o in abbinamento ad altri materiali erano d'altronde ampiamente testimoniate, sia per l'edilizia residenziale sia per quella ecclesiastica. La Vita di San Pardoux/Pardulfo racconta un episodio interessante. Il capomastro che dirigeva i lavori per la costruzione della chiesa di Saint-Aubin, a Guéret, aveva mandato i carpentieri nella foresta vicina per prendere il legname necessario, che una volta tagliato era stato caricato su un carro e trasportato al cantiere. Ma misurando le travi predisposte ci si accorse che misuravano un piede e mezzo di meno della lunghezza prevista. Il prepositus fece allora picchiare i carpentieri, ma san Pardoux/Pardulfo intervenne e operando un miracolo ristabili la misura esatta, anche superandola. Quello che vi era di troppo fu allora tagliato e sospeso nella chiesa e divenne oggetto di venerazione ${ }^{36}$. Da ricordare anche che i dipendenti del monastero di SaintGermain-des-Prés dovevano consegnare 40.00o scandolae e 20.000 axiles (piccole travi) lignee per le coperture, come segnalato dal polittico degli anni 8o6-82937.

Ma la lavorazione del legno non doveva essere legata solo all'attività edificatrice, ma anche alla produzione di suppellettili, masserizie varie, oggetti. Va poi ricordato l'utilizzo del legname per il riscaldamento, l'alimentazione dei fuochi delle cucine e del forno e degli opifici attivi in vari campi artigianali. Il problema delle attività produttive, anche quindi quelle legate alla lavorazione e messa in opera variamente del legno, costituisce un tema importante nel dibattito scientifico, coinvolgendo aspetti quali il funzionamento della realtà monastica, il rapporto tra religiosi e laici operanti a livello artigianale dentro e fuori il monastero, la partecipazione dei monasteri a tutte le fasi dei cicli produttivi, compresa la fase finale della commercializzazione.

È quindi il momento di aprire una breve parentesi sulla cultura materiale lignea legata agli ambienti monastici ${ }^{38}$. Le nostre informazioni sulla produzione diversificata di oggetti in legno si basano su testimonianze poco numerose e spesso non esaustive fornite dalle fonti scritte, oltre che da quei contesti di scavo che per la loro natura particolare (depositi di natura anaerobica, in terreni umidi o addirittura sommersi) hanno restituito elementi di questo tipo. Durante il medioevo il legno fu lavorato e usato in vari modi differenti. Basti per l'ambiente monastico procedere individuando spazi funzionali diversi. Nelle celle singole o

\footnotetext{
${ }_{32}$ Codice Diplomatico del monastero di San Colombano di Bobbio, op. cit. (n. 15).

33 Statuta antiqua abbatiae S. Petri Corbeiensis quae monachis suis praescripsit sanctus Adalhardus abbas (The Customs of Corbie- Consuetudines Corbeienses), in W. Horn, E. Born (a cura di), The Plan of St. Gall: A Study of the Architecture and Economy and Life in a Paradigmatic Carolingian Monastery, III, Berkeley-Los Angeles-London, 1979, p. 93-126; A. VERHULST, J. SEMMLER, Les statuts d'Adalhard de Corbie de l'an 822, in Le Moyen Âge, 68, 1962, p. 91-123, 233-269; J. SEMMLER, Consuetudines Corbeienses. Introductio, ed. K.Hallinger, in Corpus Consuetudinum Monasticarum, 1, Siegburg, 1963, p. 357363; D. GANZ, Corbie in the Carolingian Renaissance, Sigmaringen, 1990; F. FOUCHER, Adalhard et les deux Corbies, fondation et architecture raisonnée d'un monastère carolingien, in HAM, 13, 1, 2007, p. 75-86; F. MARAZZI, Le città dei monaci, op. cit. (n. 1), p. 258-263.

${ }^{34}$ A. GOBBI, Materiali e tecniche costruttive di un grande cantiere altomedievale, in F. Marazzi, A. Gobbi (a cura di), Il lavoro nella Regola, op. cit. (n. 25), p. $83-212$.

35 F. Marazzi (a cura di), San Vincenzo al Volturno. Guida, op. cit. (n. 12), p. 90.

${ }^{36}$ Vita Pardulf, in Acta Sanctorum, octobris, III, p. 437; Vita Pardulfi abbatis Waractensis, ed. W. Levinson, Hannoverae et Lipsiae, 1920 (MGH, Passiones vitaeque sanctorum aevi merovingicarum, Scriptores Rerum Merovingicarum, 7), p. 19-40; P. RICHÉ, La vita quotidiana nell'impero carolingio, Roma, 1994, p. 210.

${ }^{37}$ P. RICHÉ, La vita quotidiana, op. cit. (n. 36), p. 211. Per l'edizione del polittico: Polyptyque de l'abbé Irminon, ed. B. Guérard, Paris, 1844; Polyptyque de l'Abbaye de Saint-Germain-des-Prés, ed. A. Longnon, Paris, 1886-1895.

${ }^{38}$ Sulle attività produttive di tipo artigianale: F. DELL'ACQUA, Craft Production in Early Western Monasticism: Rules, Spaces, Products, in Medieval Monasticism Ante Litteram, op. cit. (n. 6), p. 289-314; E. GIANNICHEDDA, Archeologia della produzione, in S. Gelichi (a cura di), Quarant'anni di Archeologia Medievale in Italia. La rivista, i temi, la teoria e i metodi, Firenze, 2014, p. 75-94.
} 
nei dormitori comuni il letto era di legno. Nel monastero di Jumièges nella seconda metà del VII secolo il dormitorio comunitario occupava il secondo piano di un edificio e aveva $\mathrm{i}$ letti dei monaci disposti in corrispondenza di una finestra ${ }^{39}$. I refettori monastici dovevano avere dei sedili/panche $e^{40} \mathrm{e}$ dei tavoli. La Regola di Isidoro di Siviglia prevedeva che i monaci dovessero sedere due per ogni tavolo ${ }^{41}$. Potevano esserci anche delle credenze nelle quali si conservava la stoviglieria, come è documentato dagli scavi a San Vincenzo al Volturno, dove, appunto nel refettorio, sono stati ritrovati i resti carbonizzati di un grosso mobile di legno che doveva avere questa funzione ${ }^{42}$.

Molte stoviglie, oggetti per la mensa, come le posate, contenitori dovevano essere in legno, come il ligneum vasculum in cui era contenuto dell'olio «vetustate consumptum, fractisque circoli dissolutum cernerent» riaggiustato miracolosamente da San Simeone, come ci racconta il suo biografo, un anonimo benedettino, nella vita scritta poco dopo la morte del santo nel $1016^{43}$ o come le buttes e i bariles costruiti dai falegnami carpentieri bobbiesi ricordati nel Breve di Wala, insieme a scrinia ${ }^{44}$. Ma dovevano anche essere in legno manici di attrezzi da lavoro, dei quali nella maggior parte dei casi si sono conservate le sole parti in metallo. Uno spazio molto importante doveva essere quello dello scriptorium/ archivio/biblioteca, che doveva prevedere armaria per la conservazione dei libri e dei documenti. Così prescrivevano diverse Regole ${ }^{45}$ e così lascia supporre per Bobbio uno dei più antichi manoscritti bobbiesi (Amb.S.45 sup.), che reca una annotazione significativa, Liber de arca domno Atalani, con riferimento ad un armadio in cui l'abate Attala avrebbe custodito i manoscritti ${ }^{46}$.

Non dobbiamo dimenticare inoltre suppellettili ad uso liturgico e devozionale, come il vasellame in legno (poi di vetro e infine di stagno), segno di umiltà, usato inizialmente per le celebrazioni liturgiche nel monastero di Aniane, fondato da Benedetto intorno al 750 su una sua proprietà ${ }^{47}$, o come l'arca di abete, destinata a trasportare il corpo di Colombano a Pavia, così come ricordato dai Miracula, opera di un magister nella lavorazione del legno ${ }^{48}$.

A conclusione di queste riflessioni su un aspetto di quella che in altra occasione ho definito 'civiltà del legno' ${ }^{49}$, è utile ricordare le parole che Ermoldo Nigello, il Nero, fa rivolgere dai Vosgi al Reno nel poema dedicato a Ludovico il Pio e composto nel IX secolo, perfettamente esplicative e in linea con quanto detto: «è con il mio legno che si costruiscono i palazzi e le chiese; sono io che fornisco le travi di prima scelta» ${ }^{\circ}$. È il legno, in questo caso quello delle foreste dei Vosgi, che alimenta le strutture della vita quotidiana di religiosi ed anche di laici.

\footnotetext{
${ }_{39}$ Cfr. i riferimenti bibliografici alla n. 11 .

${ }^{40} \mathrm{I}$ sedili/panche in genere addossati ai muri potevano essere in muratura o in legno. Un esempio: G. CANTINO WATAGHIN, L'abbazia dei Santi Pietro e Andrea di Novalesa: il contributo delle indagini archeologiche al recupero della sua memoria, in M.G. Cerri (a cura di), Novalesa nuove luci dall'Abbazia, Milano, 2004, p. 35-57: 48-49.

${ }^{41}$ Regola di Isidoro, op. cit. (n. 7), IX, 2; G. ARCHETTI, «Sub virga magistri». Custodia e disciplina nell'educazione carolingia dei pueri oblati, in Studi medievali, terza serie, LVII, 2, 2016, p. 527-582 passim.

${ }^{42}$ F. Marazzi (a cura di), San Vincenzo al Volturno. Guida, op. cit. (n. 12), p. 82.

${ }^{43}$ P. GOLINELLI, La «Vita» di s. Simeone monaco, in Studi medievali, XX, 2, 1979, p. 709-788: XXIIII, 778.

${ }^{44}$ Codice Diplomatico del monastero di San Colombano, op. cit. (n. 15); Vedasi anche G. ARCHETTI, "De mensura potus". Il vino dei monaci nel medioevo, in Id. (a cura di), La civiltà del vino. Fonti, temi e produzioni vitivinicole dal Medioevo al Novecento, Atti della VII Biennale di Franciacorta (Monticelli Brusati - Antica Fratta, 5-6 ottobre 2001), Brescia, 2003, p. 205-326 passim.

${ }^{45}$ Regola Tarnatense, in E. Bianchi (a cura di), Regole monastiche d'Occidente, op. cit. (n.7), p. 131-153: VII, 1-2; Regola di Ferréol, in Ibid., p. 155-189: XXII, 1-2; Regola di Aureliano ai monaci, in Ibid., p. 105-129: XXVIII, 3, XXXIII e XLVIII, 3; Regola di Benedetto, in Ibid., p. XLVIII; Regola di Isidoro, in Ibid., p. VIII.

${ }^{46}$ E. DESTEFANIS, Il monastero di Bobbio, op. cit. (n. 14), p. 37, nota 38; S. LOMARTIRE, Miniatura, in A. Segagni Malacart, S. Lomartire (a cura di), Bobbio, Enciclopedia dell'arte medievale, III, Roma, 1992, p. 543-549: 543.

${ }^{47}$ ARDO SMARAGDUS, Vita Benedicti abbatis Anianensis et Indensis, ed. G.Waitz, Hannoverae, 1887 (MGH, Scriptores, XV, 1), p. 198-220: 5; G. ARCHETTI, "Dilexi decorem domus tuae", op. cit. (n. 8), p. 337-339; F. MARAZZI, Le città dei monaci, op. cit. (n. 1), p. 223.

${ }^{48}$ Miracula sancti Columbani, op. cit. (n. 26), 6, p. 1000; 9, p .1002; 27, p. 1014.

49 P. Galetti (a cura di), Civiltà del legno. Per una storia del legno come materia per costruire dall'antichità ad oggi, Bologna, 2004, p. 9-10.

${ }_{50}$ ERMOLDO IL NERO, Poème sur Louis le Pieux et épitre au roi Pépîn, ed. E. Faral, Paris, 1932, p. 209.
} 